\title{
Inv dup(22), del(22)(q11) and $r(22)$ in the father of a child with DiGeorge syndrome
}

\author{
Anne Bergman and Elisabeth Blennow
}

Department of Clinical Genetics, Karolinska Hospital, Stockholm, Sweden

\begin{abstract}
We here report a unique inherited case of DiGeorge syndrome. The asymptomatic father had a mosaic karyotype with a 21q11 deletion in three different cell lines. In two of the cell lines there was an additional supernumerary inv dup(22) or an $r(22)$, respectively. In the third cell line the del(22) was the sole anomaly. FISH analysis showed that both the inv dup(22) and the r(22) included the DGS region. We hypothesize that an inter-chromosomal recombination between inverted repeats, together with a recombination between sister chromatids during meiosis I, gave rise to a deletion of $22 q 11$ as well as an inv dup(22) containing the DGS region. The inv dup(22) was later rearranged into a ring chromosome during mitosis which was subsequently lost during cell division, thereby resulting in three different cell lines. This is the first case reported with an inv dup(22) and a del(22)(q11) in the same cell line. Our findings support a related mechanism in the formation of these two rearrangements mediated by low-copy repeats. European Journal of Human Genetics (2000) 8, 801-804.
\end{abstract}

Keywords: DiGeorge syndrome; cat eye syndrome; 22q11; deletion; inv dup(22); ring chromosome; FISH

\section{Introduction}

DiGeorge syndrome (DGS) is caused by a defect in the development of the pharyngeal arches and pouches. The major features include outflow tract malformations of the heart, a dysmorphic facial appearance, thymic hypoplasia and hypocalcaemia. ${ }^{1}$ Microdeletion of $22 q 11$ is found in about $90 \%$ of patients with DGS. The deletion is inherited in approximately $20 \%$ of the cases and it is therefore recommended to screen parents for carrier status. Carrier parents frequently appear to be more mildly affected than their children. The fact that most DGS cases occur de novo indicates that the 111 region on chromosome 22 is especially prone to rearrangements.

Cat eye syndrome (CES) is another, less frequent $22 q 11$ disorder with a variable phenotype including ocular coloboma, anal atresia, heart defects and dysmorphic features. ${ }^{2}$ CES is associated with a duplication 22 pter $\rightarrow 22$ q11, most often in the form of a supernumerary marker chromosome, an inv dup(22). The marker chromosome is reported to vary in size with some of the CES chromosome breakpoints corresponding to common breakpoints of the DGS deletion.

Correspondence: Dr Elisabeth Blennow, Department of Clinical Genetics, Karolinska Hospital, S171 76 Stockholm, Sweden. Tel: +468517 75380; Fax: +468327734; E-mail: ebl@gen.ks.se

Received 25 February 2000; revised 9 May 2000; accepted 18 May 2000
This suggests that the mechanism of formation of the inv dup(22) and the del(22)(q11) may be related. ${ }^{3,4}$

We here describe a child with DGS due to an inherited $22 q 11$ deletion. The father has a complex mosaic karyotype that includes a $22 q 11$ deletion as well as a $22 q 11$ duplication in the form of an inv dup(22) or a r(22).

\begin{abstract}
Materials and methods
Case history

The proband, the first son of healthy and unrelated parents, was born at term after an unremarkable pregnancy, with a birth weight of $3275 \mathrm{~g}$ and a birth length of $51 \mathrm{~cm}$. Due to breathing difficulties at 2 days old, cardiac investigation with echography and angiography was performed and showed an interrupted aortic arch and a ventricular septum defect. He also had borderline hypocalcaemia and a dysmorphic facial appearance reminiscent of DGS.
\end{abstract}

\section{Cytogenetic analysis}

Peripheral blood samples were obtained from the proband, and subsequently from his parents and his paternal grandparents. M etaphase chromosome spreads were prepared from 


\section{G-banding}

\section{LSI TUPLE1 SpO/ LSI ARSA SpG}

I

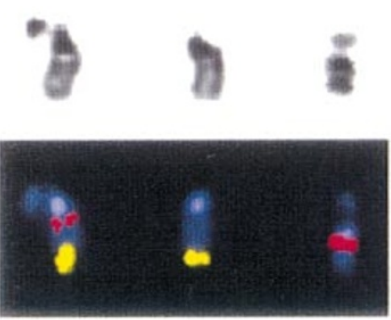

II

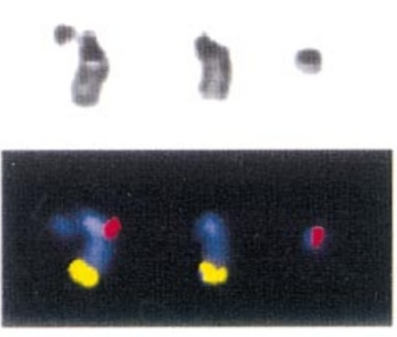

III

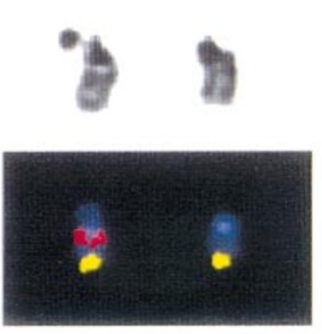

Figure 1 Mosaicism with three different cell lines. The G-banded chromosomes on top and the FISH results using LSI TUPLE1 SpO/LSI ARSA SpG below.

lymphocyte cultures and routine GTG-banding was performed according to standard procedures.

\section{FISH}

The probe LSI TUPLE1(22q11, DGS region) Spectrum Orange/LSI ARSA(22q11, control probe) Spectrum Green (Vysis, Downers Grove, IL, USA) was used according to the manufacturer's instructions. The probe alpha XT680 (centromere14/22 specific probe) was labelled with FITC-
12-dUTP (Vector Lab, Burlingame, LA, USA) by nick translation and used as previously described. ${ }^{5}$

\section{Results}

FISH analysis using the probe LSI TUPLE1/LSI ARSA showed a microdeletion of $22 q 11$ in the child, thus confirming the DGS diagnosis. Chromosomal and FISH analyses of the mother and the paternal grandparents were all normal. Cytogenetic analysis of the apparently asymptomatic and

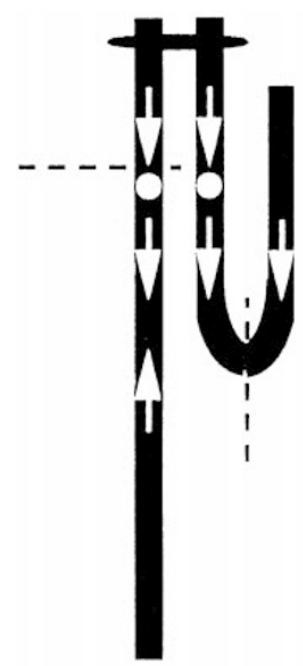

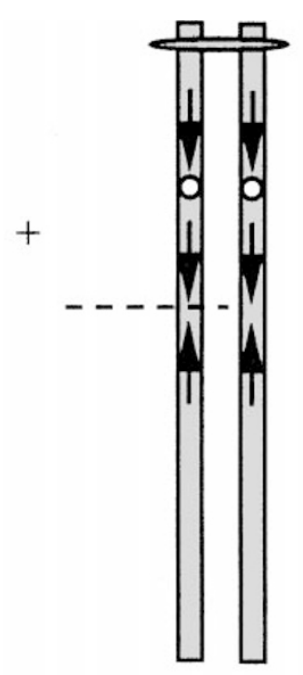

centromere

O DGS region
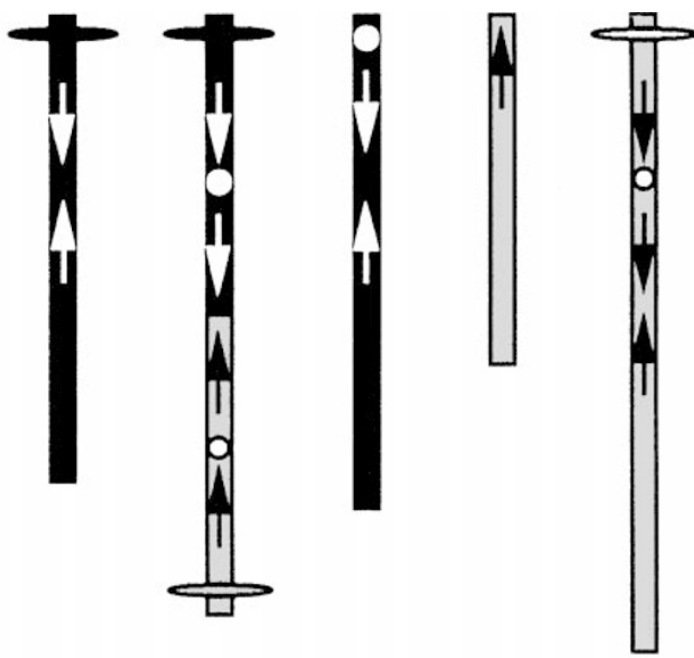

del

inv dup ace

ace

Figure 2 Inter-chromosomal recombination between inverted repeats combined with a recombination between sister chromatids resulted in a del(22)(q11) and an inv dup(22). 
non-dysmorphic father revealed mosai cism with three different cell lines (Figure1). FISH analysis using LSI TUPLE1/LSI ARSA showed a deletion of TUPLE1 in one of the cytogenetically normal chromosomes 22 in all three cell lines. In the first cell line ( $12 \%$ of the cells according to $\mathrm{FISH}$ analysis), there was also a supernumerary marker chromosome. Since the marker chromosome showed labelling with TUPLE1 but not with ARSA and double labelling with the 14/22 centromere specific probe, it was identified as an inv dup(22). The second cell line ( $23 \%$ of the cells) had a supernumerary ring chromosome in addition to the deleted chromosome 22. The ring chromosome showed labelling with TUPLE1 but not with ARSA and single labelling with the 14/22 centromere specific probe. The third cell line (65\% of the cells) had deletion of $22 q 11$ as the sole anomaly. In conclusion, the father's karyotype was:

$$
\begin{aligned}
& \text { 46,XY, del(22)(q11q11)/47,idem, +r(22)(p?p?)/47,idem, } \\
& + \text { inv dup(22)(pter } \rightarrow \text { q11::q11 } \rightarrow \text { pter) }
\end{aligned}
$$

\section{Discussion}

Mosaicism for structural aberrations is rare and the presence of different rearrangements involving the same chromosomal region is intriguing. We have found the simultaneous occurrence of a del(22)(q11) and an extra chromosome containing the $22 q 11$ region, suggestive of a common

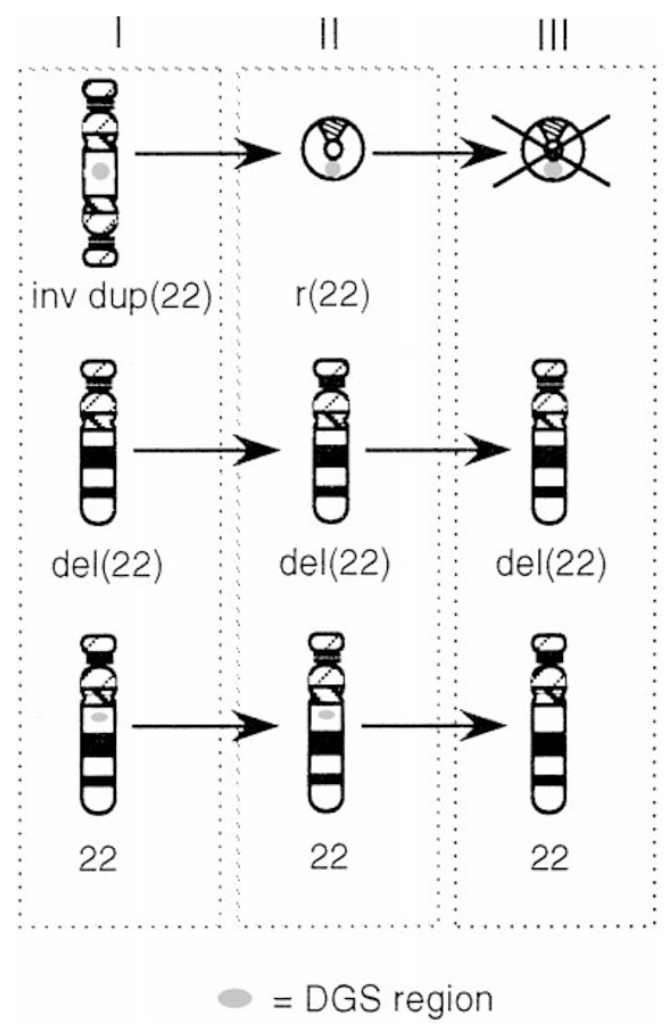

Figure 3 The evolution of the three different cell lines of the father. mechanism of formation of these rearrangements. The fact that low-copy repeats on $22 \mathrm{q} 11$ may mediate rearran gements both for CES chromosomes and 22q11 deletions is consistent with a related origin. ${ }^{6}$

Microdeletions are considered to arise through unequal crossing over as the result of misalignment in homologous regions. ${ }^{7}$ Low-copy-number repeat sequences flank the DGS region at $22 q 11,{ }^{8}$ and recent studies by Edelman et al have shown that the breakpoints of the common DGS deletion on $22 q 11$ are located in those highly homologous low-copy repeats. ${ }^{9}$ The formation of a deletion may also result in a reciprocal duplication product. This is thought to be the case in Charcot-Marie Tooth disease 1A and HNPP (hereditary neuropathy with pressure palsies), where the structural rearrangement is a duplication or a deletion of the same region on $17 \mathrm{p} .{ }^{10}$ Edelman et al described a family with an interstitial duplication of the $3 \mathrm{Mb}$ region that is deleted in DGS patients. ${ }^{9}$ To the best of our knowledge, a $22 q 11$ duplication together with a deletion in the same patient has hitherto never been reported.

Inverted duplications have been suggested to arise as a U-type exchange between homologous chromosomes ${ }^{11,12}$ or inter-chromosomal recombination between inverted sequences. ${ }^{6,13}$ Another alternative is repair of a broken chromosome through replication and end to end fusion. ${ }^{11}$

We hypothesize that an inter-chromosomal recombination between inverted repeats combined with a recombination between sister chromatids during meiosis I gave rise to both a deleted 22q11 and an inv dup(22) containing the DGS region (Figure2). The acentric fragments were lost. After fertilization the cell line included one normal chromosome22, one deleted chromosome22 and the inv dup(22). The inv dup(22) was later rearranged into a ring chromosome which, due to its unstable nature, was subsequently lost during mitotic cell division, thereby giving rise to three different cell lines (Figure3).

A few cases with inv dup chromosomes and a del etion or a duplication of the homologous regions have previously been reported. Spinner et al described a patient with Angelman syndrome due to a deletion of one of the cytogenetically normal chromosomes 15 and an inv dup(15) that, in contrast to our case, did not include the deleted region. ${ }^{14}$ Therefore, the inv dup(15) was not believed to contribute to the patient's phenotype. It was speculated that patients with inv dup(15)s may be at increased risk for other abnormalities involving 15q11-q13 and it was suggested that when one cytogenetic abnormality occurred, it could predispose to a second rearrangement during meiosis. Leana-Cox et al described a patient with an inv $\operatorname{dup}(15)$ and a dup(15), ${ }^{15}$ and a similar finding was made by Abeliovich et al. ${ }^{16}$ Mears et al reported the presence of an interstitial duplication of $22 q$ in combination with an inv dup(22). ${ }^{17}$ However, the present case is the first to be described with an inverted duplication and a deletion involving the same region on $22 \mathrm{q}$. 
The risk of recurrence of DGS in our case is difficult to estimate due to the presence of mosaicism and many possible modes of segregation. The risk of DGS could be as high as $50 \%$. In addition, there is also a theoretical risk of offspring with cat eye syndrome if the inv $\operatorname{dup}(22)$ or the $r(22)$ is inherited together with a normal chromosome22. Prenatal diagnosis may be offered to the couple, including chromosome analysis and FISH after chorionic villus sampling or amniocentesis.

The cytogenetic findings in this case provide a possible link between DGS and CES. Future similar cases will hopefully further elucidate the mechanism of formation of these structural rearrangements.

\section{Acknowledgements}

Wethank DrThe-Hung Bui for critical reading of the manuscript. This work was supported by the Swedish Medical Research Council and Magnus Bergvalls Stiftelse.

\section{References}

1 Wilson DI, Burn J, Scambler P, Goodship J: DiGeorge syndrome: part of CATCH 22. J Med Genet 1993; 30: 852-856.

2 Schinzel A, Schmid W, Fraccaro $M$ et al: The 'cat eye syndrome': dicentric small marker chromosome probably derived from a no: 22 (tetrasomy 22pter to q11) associated with a characteristic phenotype. Hum Genet 1981; 57: 148-158.

3 CrollajA, Howard P, Mitchell C, Long FL, Dennis NR: A molecular and FISH approach to determining karyotype and phenotype correlations in six patients with supernumerary marker(22) chromosomes. Am J Med Genet 1997; 72: 440-447.

4 McTaggart KE, Budarf ML, Driscoll DA, Emanuel BS, Ferreira P, McDermid HE: Cat eye syndrome chromosome breakpoint clustering: identification of two intervals also associated with 22q11 deletion syndrome breakpoints. Cytogenet Cell Genet 1998; 81: 222-228.

5 Blennow E, Anneren G, Bui TH, Berggren E, Asadi E, Nordenskjöld $\mathrm{M}$ : Characterization of supernumerary ring marker chromosomes by fluorescence in situ hybridization (FISH). Am J Hum Genet 1993; 53: $433-442$.
6 Edelman L, Pandita RK, Spiteri E et al: A common molecular basis for rearrangement disorders on chromosome22q11. Hum Mol Genet 1999; 8: 1157-1167.

7 Chen KS, Manian P, Koeuth T et al: Homologous recombination of a flanking repeat gene cluster is a mechanism for a common contiguous gene deletion syndrome. Nat Genet 1997; 17: 154-163.

8 Halford S, Lindsay E, Nayudu M, Carey AH, Baldini A, Scambler PJ: Low-copy-number repeat sequences flank the DiGeorge/velocardio-facial syndrome loci at 22q11. Hum Mol Genet 1993; 2: 191-196.

9 Edelman L, Pandita RK, M orrow BE: Low-copy repeats mediate the common 3-Mb deletion in patients with velo-cardio-facial syndrome. Am J Hum Genet 1999; 64: 1076-1086.

10 Chance PF, Abbas N, Lesch MN et al: Two autosomal dominant neuropathies result from reciprocal DNA duplication/deletion of a region on chromosome17. Hum Mol Genet 1994; 3: 223-228.

11 Schreck RR, Breg WR, Erlanger BF, Miller OJ: Preferential derivation of abnormal human G-group-like chromosomes from chromosome15. Hum Genet 1977; 36: 1-12.

12 Van Dyke DL, Weiss L, Logan M, Pai GS: The origin and behavior of two isodicentric bisatellited chromosomes. Am J Hum Genet 1977; 29: 294-300.

13 Robinson WP, Dutly F, Nicholls RD et al: The mechanisms involved in formation of deletions and duplications of 15q11-q13. J Med Genet 1998; 35: 130-136.

14 Spinner NB, Zackai E, Cheng S-D, Knoll JH: Supernumerary inv dup(15) in a patient with Angelman syndrome and a deletion of 15q11-q13. Am J Med Genet 1995; 57: 61-65.

15 Leana-Cox J, Jenkins L, Palmer CG et al: Molecular cytogenetic analysis of inv dup(15) chromosomes, using probes specific for the Prader-Willi/Angelman syndrome region: clinical implications. Am J Hum Genet 1994; 54: 748-756.

16 Abeliovich D, Dagan J, Werner M, Lerer I, Shapira Y, Meiner V: Simultaneous formation of inv dup(15) and dup(15q) in a girl with developmental delay: origin of the abnormal chromosomes. Eur J Hum Genet 1995; 3: 49-55.

17 Mears AJ, Duncan AM, Budarf ML et al: Molecular characterization of the marker chromosome associated with cat eye syndrome. Am J Hum Genet 1994; 55: 134-142. 